\title{
SYSTEM IDENTIFICATION AND HSDBC-OPTIMIZED PID CONTROL OF A PORTABLE LOWER-LIMB REHABILITATION DEVICE
}

\author{
SULAIMAN O. FADLALLAH ${ }^{1}$, KHALED M. GOHER ${ }^{2}$ \\ Auckland University of Technology,NZ,e-mail: sulaiman.fadlallah@aut.ac.nz \\ University of Lincoln, UK, e-mail: kgoher@lincoln.ac.uk
}

\begin{abstract}
The present paper introduces a novel portable leg rehabilitation system (PLRS) that is developed to provide the user with the necessary rehabilitation exercises for both the knee and ankle in addition to the portability feature to overcome the hardships associated with both effort and cost of hospitals and rehabilitation clinics' steady sessions. Prior realizing the actual prototype, the proposed configuration was visualized using SolidWorks including its main components. Aiming to control the developed system, and given the fact that tuning controller parameters is not an easy task, Hybrid Spiral-Dynamics Bacteria-Chemotaxis (HSDBC) algorithm has been applied on the proposed control strategy in order to obtain a satisfactory performance. The obtained system performance was satisfactory in terms of desired elevation and settling time.
\end{abstract}

\section{Introduction}

Assistive robotic technology for rehabilitation has been and still remains a major field of interest for tremendous amount of research studies over the past years. Rehabilitation devices can be divided into five different categories: stationary gait trainers, treadmill gait trainers, ankle rehabilitation devices, over-ground gait trainers, and foot-plate-based gait trainers [1]. Focusing on lower-limb rehabilitation systems, considerable amount of studies have been conducted in this area. Starting with Hwang and Jeon [2], their research focused on developing a wheelchair integrated lower limb exercise/rehabilitation system. The system consists of a wheelchair, a body lifter, and a lower-limb exoskeleton. The research studies carried out by Banala et al. [3], Agrawal et al. [4] and Banala et al. [5] aimed gradually to design a gravity-balancing exoskeleton with the name ALEX. As for Monaco et al. [6], their study presented a stationary gait trainer (NEUROBike) for bedridden patients that provides them with neuro-rehabilitative treatments. On the other hand, and by utilizing MATLAB ${ }^{\circledR} /$ Simulink software, Zhang and Li [7] proposed a lowerlimb rehabilitation mechanism with 4-degrees of freedom. Given the fact that these lower-limb rehabilitation devices must consider the user's physical and health conditions, they require a proper control in order to provide smooth and comfortable operation of the leg movement. Focusing on controlling the cyclical leg movements of paraplegic subjects, Spek et al. [8] developed a control scheme that combines both neural network and fuzzy logic. As for AguirreOllinger et al. [9], their study focused on developing an inertia compensationbased control scheme for a one degree of freedom exoskeleton that assists patients with lower-limb disabilities. Tuning controllers is a difficult problem that remains a field of interest in multiple disciplines. This interest drove 
researcher worldwide to provide solutions and techniques for optimizing controller parameters. In nowadays research aspects, bio-inspired and natureinspired optimization algorithms are significantly gaining attention. Some of these algorithms include genetic algorithm (GA) [10], bacterial foraging optimization algorithm (BFO) [11], spiral dynamics algorithm (SDA) [12], and Hybrid Spiral-Dynamics Bacteria-Chemotaxis (HSDBC) algorithm [13] that represents a fusion between the latter two algorithms. Although the field of lower-limb rehabilitation devices has been provided a lot of interest, up to the authors' knowledge no research addressed the portability feature in developing an affordable portable lower-limb rehabilitation device that gives the user the flexibility needed in performing rehabilitation exercises. In this paper, the authors aim at developing a novel portable leg rehabilitation system (PLRS) that provides the user with the necessary extension/flexion exercises for both the knee and ankle in addition to the portability feature to overcome the hardships associated with both effort and cost of hospitals and rehabilitation clinics' steady sessions. The presented work in this paper is arranged as follows: Section I presents an overview of contributions in the field of lower-limb rehabilitation systems along with their control methods and the potential of optimization algorithms. In Section II, the proposed portable leg rehabilitation system (PLRS) is presented, starting with its concept schematics, moving to the SolidWorks design, and ending with the actual prototype. Section III focuses on the process of estimating the prototype's transfer functions by utilizing System Identification (SI). The design of the control approach including the implementation of HSDBC optimization algorithm is covered in Section IV. Finally, and by highlighting the research findings, the paper is concluded in Section V.

\section{Portable Leg Rehabilitation System Description and Design Details}

\subsection{System Description}

Fig. 1 illustrates the developed portable leg rehabilitation system (PLRS) schematics diagram. The proposed two degrees of freedom system consists of two movable links $\left(r_{1}\right.$ and $\left.r_{2}\right)$ connected with two revolute joints.

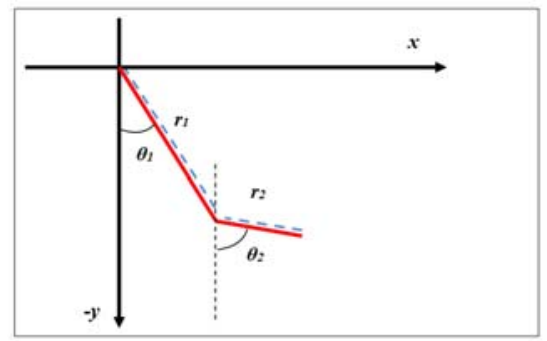

Figure 1. Schematics diagram of the proposed PLRS 
By applying a lifting torque at each joint, the system has the capability to carry out three different rehabilitation exercises by lifting the targeted link to a desired position $(\theta)$ (i.e. calf exercise $\left(\theta_{l}\right)$, foot exercise $\left(\theta_{2}\right)$, both calf and foot exercises $\left(\theta_{1}\right.$ and $\left.\left.\theta_{2}\right)\right)$.

\subsection{Design Details}

Taking into account the targeted portability feature, and based on the schematics illustrated in Fig. 1, a SolidWorks conceptual design of the PLRS is demonstrated in Fig. 2. The proposed design main components are calf and foot carriers, thigh covers, and thigh and seat bases. The grooves in both seat and thigh bases simplifies the device adjustments based on the user's requirement. Fig. 3 represents the realized design of the portable device. The system's chassis was manufactured from aluminum to provide a lightweight structure. Two motors are mounted at each joint in order to provide the necessary lifting torque on the calf and foot carriers. These motors are controlled using the circuit shown in Fig.4. The potentiometer's produced analog signal is responsible for controlling the motor's speed and orientation. Given that the speed control's analog voltage range is within $0-5$ volts DC, three voltage values are considered, $3.1 \mathrm{~V}$ (lifting), $2.5 \mathrm{~V}$ (zero speed), and 1.9V (descending).

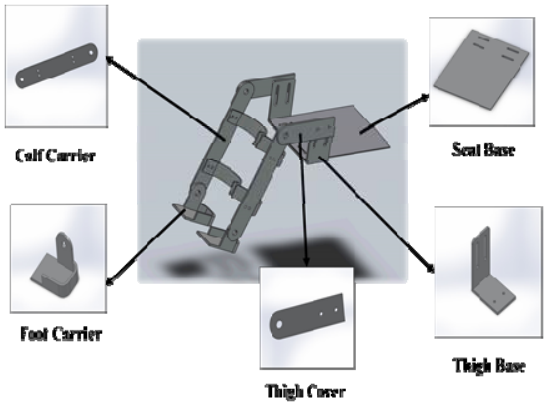

Figure 2. PLRS SolidWorks design

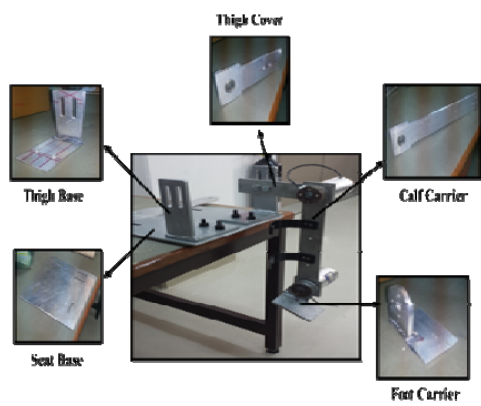

Figure 3. The developed PLRS.

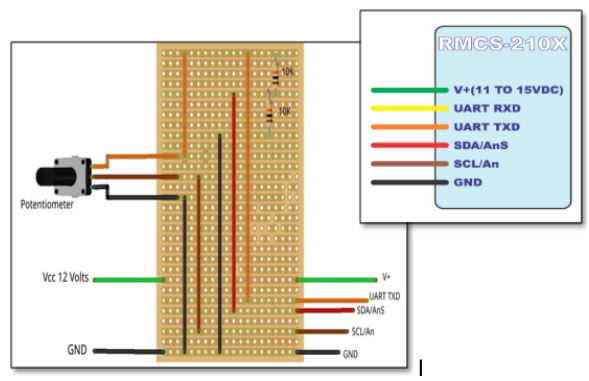

Figure 4. PLRS motor control circuit 


\section{System Identification (SI)}

\subsection{Overview of $S I$}

By utilizing measurements of the device's input and output signals, SI is considered a powerful approach for deriving mathematical representations of dynamic systems. A graphical user interface (GUI) developed by Matlab (Fig. 5) allows the user to simply estimate the system's model by inserting the measured data and adjusting certain parameters until the estimated result becomes closer to the measured output signal. In this part of the study, the PLRS's transfer functions estimation will be carried out based on measured input and output signals extracted from the built experimental setup (Fig. 3).

\subsection{Estimation of Calf and Foot Transfer Functions}

The prototype's system identification process is divided into three major parts: analog input measurements, analog output measurements, and transfer function estimation. For this system, the variation in voltage from the potentiometer is considered as an analog input. The system's outputs are both calf and foot orientations. These orientations are measured using a position sensor and its output is processed using Humosoft MF624 data acquisition card connected to Matlab/Simulink environment.

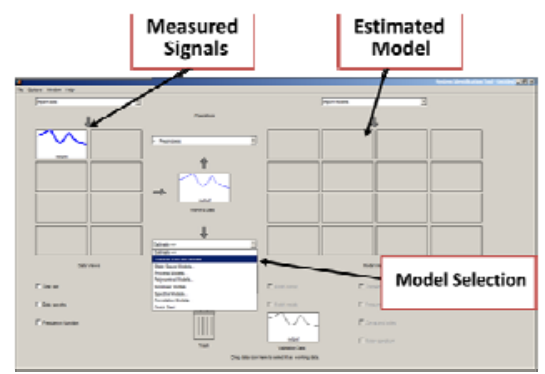

Figure 5. Matlab’s SI toolbox GUI

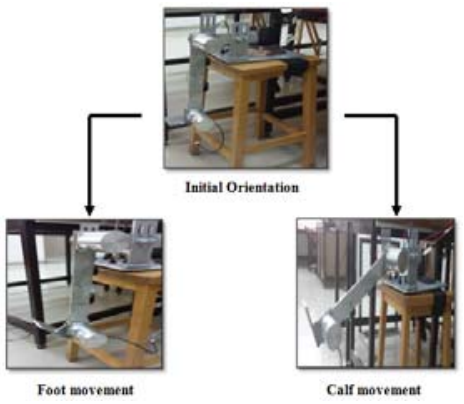

Figure 6. PLRS rehabilitation exercises

Two rehabilitation exercises are considered and are shown in Fig. 6: calf exercise only $\left(\theta_{1}\right)$ and foot exercise only $\left(\theta_{2}\right)$. The system considered to orient the calf by 90 degrees $\left(\theta_{1}=90^{\circ}\right)$ and the foot by 30 degrees $\left(\theta_{2}=30^{\circ}\right)$. Referring to Fig. 7, which represents the calf and foot orientation's measured output, the rehabilitation process is split into three major stages: (1) extension, (2) stability, and (3) flexion. For the calf movement, the actual prototype's maximum measured position was approximately 86 degrees $\left(\theta_{l}=86^{\circ}\right)$ and it takes around 12 seconds to reach this position. As for the foot, the maximum measured angular orientation was approximately 32 degrees $\left(\theta_{l}=32^{\circ}\right)$ and the system takes approximately 5 seconds to reach this desired position. These measured 
outputs and the potentiometer's input are considered as inputs to the Matlab's SI toolbox. In order to estimate the system's appropriate transfer functions, the desired number of poles and zeros are also fed into the toolbox. Two combinations of poles and zeros are considered in order to acquire the best fit from the measured date. A comparison between the measured outputs and the two estimated models for both calf and foot is illustrated in Fig. 8.

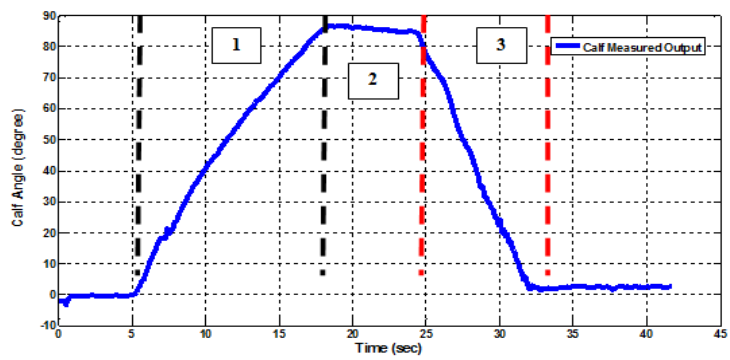

(a) Calf exercise

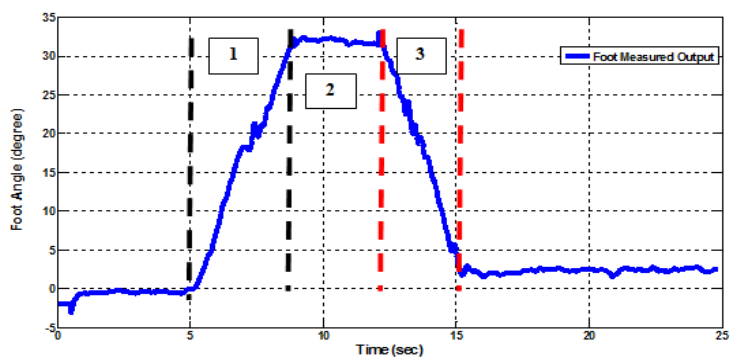

(b) Foot exercise.

Figure 7. Measured outputs of calf and foot's orientation. 


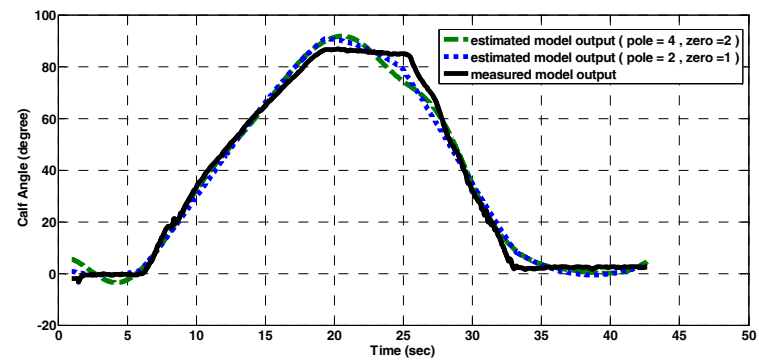

(a) Calf exercise

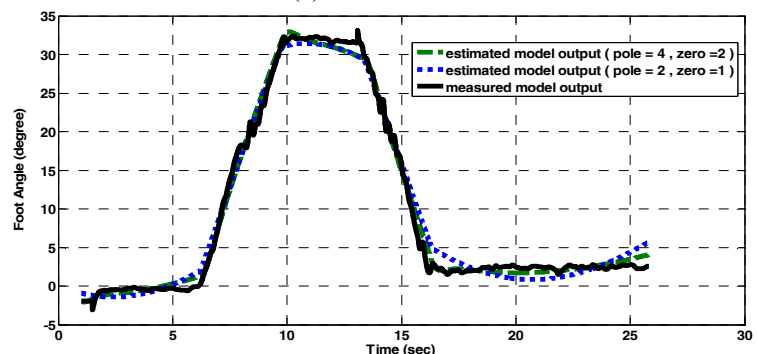

(b) Foot exercise

Figure 8. Measured and estimated model output for calf and foot

Starting with the calf, the combination of two poles and one zero provides better fitting (90.28\%) compared to the estimation with the combination of four poles and two zeros (89.89\%). As for the foot, on the other hand, the four polestwo zeros model gives better fitting (92.75\%) in comparison with the combination of two poles and one zero (88.87\%). Based on that, the estimated transfer functions for the calf and foot are represented as the following:

Calf Transfer Function $=\frac{1.449 s+0.6824}{s^{2}+0.1549+0.02833}$

Foot Transfer Function $=\frac{65.63 s^{2}+174 s+0.9918}{s^{4}+5.659 s^{3}+13.38 s^{2}+0.6049 s+0.2041}$

\section{Control System Design}

For the purpose of obtaining a satisfactory response for the system's transfer functions, this section focuses on proposing a proper control approach by implementing Hybrid Spiral-Dynamics Bacteria-Chemotaxis (HSDBC) algorithm, which is a recently developed optimization algorithm that combines both bacterial foraging and spiral dynamics algorithms. The HSDBC optimization algorithm's main parameters and flowchart are shown in Table 1 
and Fig 9. Additional information can be found in [13]. Fig. 10 demonstrates the designed HSDBC-optimized PID control approach for controlling the calf and foot's angular orientation. The optimization process was constrained within specific boundary limits and the performance index has been selected based on the minimum mean squared error (MSE). For each control loop shown in Fig. 10, the MSE can be calculated using the following equations:

Objective Function $1=\min \left[\frac{1}{N} \sum_{i=1}^{N}\left(\theta_{1 d}-\theta_{1 m}\right)^{2}\right]$

Objective Function $2=\min \left[\frac{1}{N} \sum_{i=1}^{N}\left(\theta_{2 d}-\theta_{2 m}\right)^{2}\right]$

Based on the total MSE, the PLRS's total objective function can be calculated using the following mathematical representation:

$\mathrm{J}=\sum_{i=1}^{J}($ Objective Function i)

Table 1. Parameters Associated with HSDBC Optimization Algorithm [13]

\begin{tabular}{|c|l|}
\hline Parameter & \multicolumn{1}{|c|}{ Description } \\
\hline$\theta_{i, j}$ & Bacteria angular displacement on $\mathrm{x}_{\mathrm{i}}-\mathrm{x}_{\mathrm{j}}$ plane around the origin \\
\hline$R$ & Spiral radius \\
\hline$m$ & Number of search points \\
\hline$k_{\max }$ & Maximum iteration number \\
\hline$N_{s}$ & Maximum number of swim \\
\hline$x_{i}(k)$ & Bacteria position \\
\hline$R^{n}$ & $\mathrm{n}$ x n matrix \\
\hline
\end{tabular}




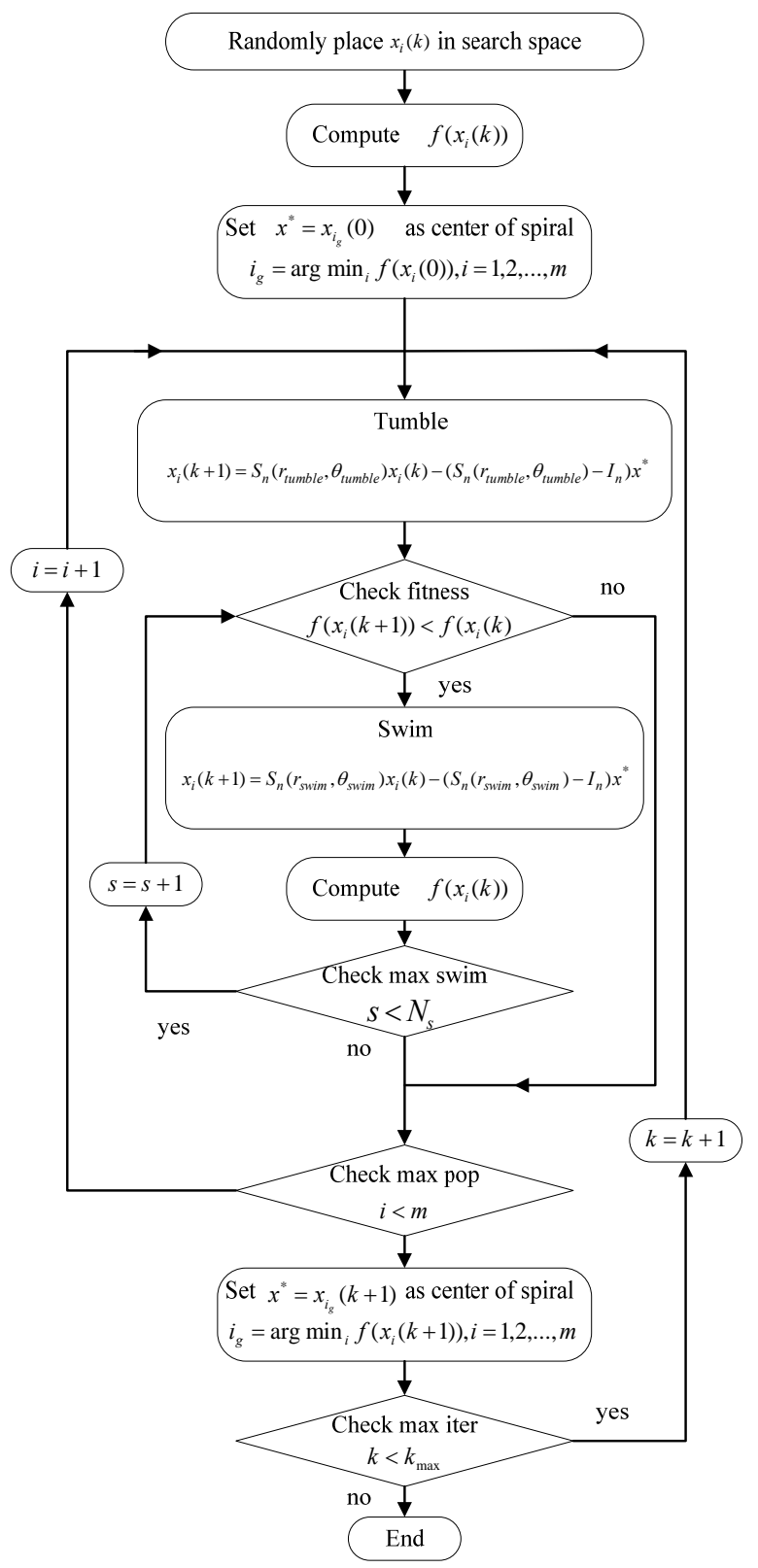

Figure 9. HSDBC algorithm flowchart 


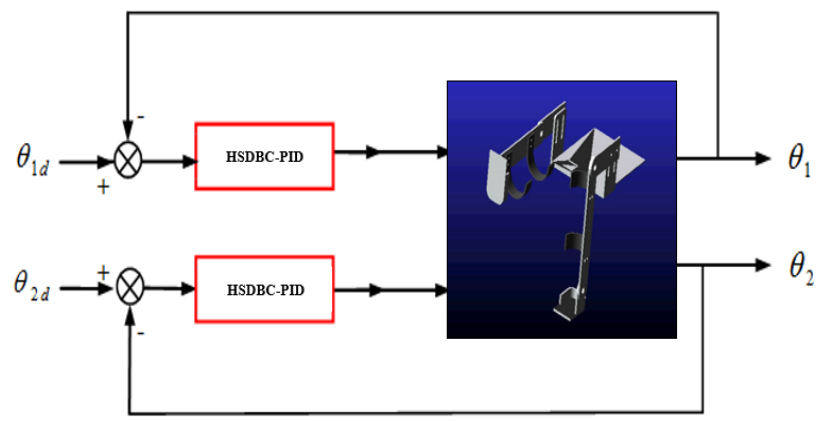

Figure 10. PLRS control scheme.

\subsection{Simulation Results}

Table 2 lists the calculated optimized controller gain parameters. Fig. 11 illustrates the system response and Table 3 summarizes the performance of the system for both calf and foot using the proposed control scheme. As can be seen, the system reach the desired orientation for both calf and foot. Starting with the results associated with the calf, and with no observable overshoot, the system managed to reach the desired elevation $\left(\theta_{1}=89.9094^{\circ}\right)$ and it takes around 12 seconds to settle. Moving to the foot's simulation results, the system settles at an angle of 30.017 degrees, which is approximately $0.056 \%$ greater than the foot's required orientation. The system's response, with an overshoot of approximately $0.53 \%$, takes approximately 6 seconds to stabilize.

Table 2. HSDBC-PID Controller Gain Parameters

\begin{tabular}{|c|c|c|}
\hline & $\begin{array}{c}\text { Gain } \\
\text { Parameter }\end{array}$ & HSDBC-PID \\
\hline \multirow{3}{*}{$\underset{\widetilde{E}}{\overparen{E}}$} & $K p_{1}$ & 0.401 \\
\hline & $K i_{1}$ & 0.010 \\
\hline & $K d_{1}$ & 1.398 \\
\hline \multirow{3}{*}{ 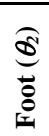 } & $K p_{2}$ & 3.012 \\
\hline & $\overline{K i_{2}}$ & 0.010 \\
\hline & $K d_{2}$ & 5.103 \\
\hline
\end{tabular}




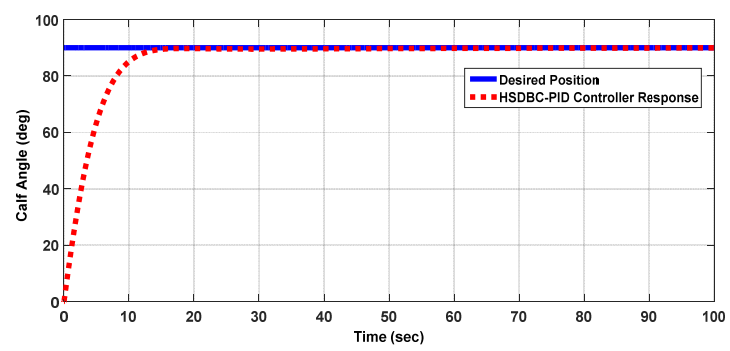

(a) Calf system response.

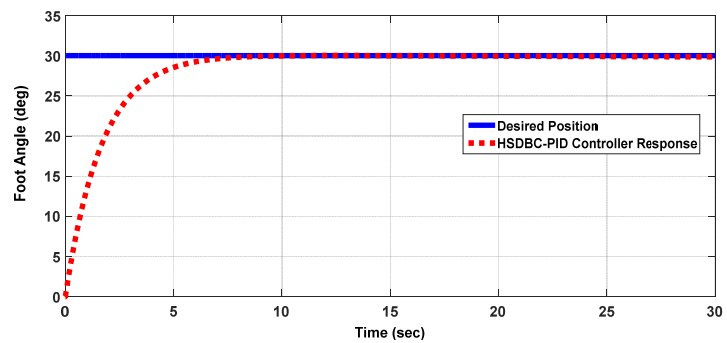

(b) Foot system response

Figure 11. Calf and foot system response using HSDBC-PID controller.

Table 3. System Performance Characteristics

\begin{tabular}{|c|l|l|c|l|l|}
\hline Variable & Parameters & \multicolumn{1}{|c|}{ Values } & Variable & Parameters & \multicolumn{1}{|c|}{ Values } \\
\hline \multirow{6}{*}{$\theta_{1}$} & Rise Time & 7.8159 & & Rise Time & 3.5904 \\
& Settling Time & 12.3080 & & Settling Time & 6.0497 \\
& Settling, Min & 80.9262 & & Settling, Min & 26.8766 \\
& Settling, Max & 89.9094 & \multirow{2}{*}{$\theta_{2}$} & Settling, Max & 30.0177 \\
& Overshoot & 0 & & Overshoot & 0.5393558 \\
& Undershoot & 0 & & Undershoot & 0 \\
& Peak & 89.9094 & & Peak & 30.0177 \\
& Peak Time & 100.000 & & Peak Time & 12.5039 \\
\hline
\end{tabular}

\section{Conclusion}

Aiming to provide an affordable portable lower-limb rehabilitation device that gives the user the flexibility needed in performing rehabilitation exercises, this paper presents a novel portable leg rehabilitation system (PLRS) that provides the user with the necessary extension/flexion exercises for both the knee and ankle. The portability feature provided by the device will overcome the hardships associated with both effort and cost of hospitals and rehabilitation clinics' steady sessions. Before actualizing the PLRS, the proposed system has 
been described and designed using SolidWorks. The experimental setup has been built for the purpose of estimating its transfer functions using system identification toolbox. For controlling the developed system's transfer functions, HSDBC optimization technique has been implemented on a proposed PID control scheme. Using the proposed control approach, the obtained system performance was satisfactory except for the very small overshoot that the system experienced for the foot movement. Future considerations of this work will include design improvements and developing a mathematical model based on the PLRS's schematics and compare its results with the actual prototype's estimated transfer functions. Future work will consider also implementing other optimization techniques (i.e. bacterial forging (BF), genetic algorithm (GA), spiral dynamics (SD)) and compare between them in order to improve the performance of the system.

\section{References}

1. I. Díaz, J. J. Gil, and E. Sánchez, "Lower-Limb robotic rehabilitation: Literature review and challenges,” Journal of Robotics, pp. 1-11, 2011.

2. B. Hwang and D. Jeon, "A wheelchair integrated lower limb exercise/rehabilitation system: Design and experimental results on the knee joint,” Proceedings of the 2012 IEEE/SICE International Symposium on System Integration (SII), Kyushu University, Fukuoka, Japan, 2012, pp. 164-169.

3. S. K. Banala, S. K. Agrawal, A. Fattah, V. Krishnamoorthy, W.-L. Hsu, J. Scholz, and K. Rudolph, "Gravity-Balancing leg orthosis and its performance evaluation,” IEEE Transactions on Robotics, vol. 22, no. 6, pp. 1228-1239, 2006.

4. S. K. Agrawal, S. K. Banala, A. Fattah, V. Sangwan, V. Krishnamoorthy, J. P. Scholz, and W.-L. Hsu, "Assessment of motion of a swing leg and gait rehabilitation with a gravity balancing exoskeleton," IEEE transactions on neural systems and rehabilitation engineering, vol. 15, no. 3, pp. 410-20, 2007.

5. S. K. Banala, S. H. Kim, S. K. Agrawal, and J. P. Scholz, "Robot assisted gait training with active leg exoskeleton (ALEX)," IEEE Transactions on Neural Systems and Rehabilitation Engineering, vol. 17, no. 1, pp. 2-8, 2009.

6. V. Monaco, G. Galardi, M. Coscia, D. Martelli, and S. Micera, "Design and evaluation of NEUROBike: A neurorehabilitative platform for bedridden post-stroke patients," IEEE transactions on neural systems and rehabilitation engineering, vol. 20, no. 6, pp. 845-52, 2012.

7. M. Zhang and X. Li, “A design of the mechanism for lower limbs passive rehabilitation and kinematics analysis," 2010 Fifth International 